\title{
Relaxação via Barreira Logarítmica Modificada Aplicada ao Problema de Fluxo de Potência Ótimo CC com Sobrecargas
}

\author{
Mayk V. Coelho, \\ UNIFAL-MG - Instituto de Ciência e Tecnologia \\ Campus Poços de Caldas \\ 37715-400, Poços de Caldas, MG \\ E-mail: mayk.coelho@unifal-mg.edu.br.
}

\author{
Anésio S. Júnior, \\ UNICAMP - Depto de Sistemas \\ 113083-852, Campinas, SP \\ E-mail: anesio@densis.fee.unicamp.br,
}

\author{
Aurélio L. Oliveira, \\ Depto de Matemática Aplicada \\ 13083-859, Campinas, SP \\ E-mail: aurelio@ime.unicamp.br
}

Resumo: Os métodos de pontos interiores apresentam bons resultados na resolução de problemas de fluxo de potência ótimo CC, porém, podem sofrer problemas numéricos quando variáveis de decisão permanecem, por diversas iterações, próximas a valores de fronteira, influenciando na convergência do método. Estes erros numéricos ocorrem com mais frequência quando há sobrecargas de geração e/ou transmissão no sistema. Estas sobrecargas podem ocasionar blackouts devido a incapacidade de satisfazer a demanda ou mesmo de transmitir a carga gerada, ocasionando em um problema infactivel.

A fim de eliminar estas dificuldades destes métodos, é proposta uma troca de função barreira, substituindo a barreira logarítmica clássica pela modificada. Esta mudança permite que haja violações controladas em algumas restriçôes de desigualdade, permitindo o uso em problemas de fluxo de potência com sobrecargas. Estudos de casos são feitos simulando situações normais e de sobrecargas no sistema IEEE-30.

Palavras-chave: Barreira Logarítmica Modificada; Pontos Interiores; FPO; Sobrecarga;

\section{Introdução}

O modelo de fluxo de potência ótimo (FPO) tem aplicações em diversos problemas de análise e operação de sistemas de potência, tais como despacho econômico, análise de confiabilidade do sistema, análise de segurança, programação da geração em curto prazo e planejamento de expansão do sistema geração/transmissão.

Em diversas aplicações tem sido adotada a representação linearizada $(\mathrm{CC})$ devido à maior simplicidade e ao grau de precisão satisfatório de seus resultados, podendo ser formulada como um modelo de fluxo em redes com restrições adicionais $[2,4,1]$.

Uma alternativa viável para resolução de problemas de FPO são os métodos de pontos interiores, por serem numericamente robustos e independem de ajustes de parâmetros para cada problema, entretanto, podem apresentar erros numéricos quando variáveis de decisão ficam muito próximas de valores de fronteira por diversas iterações.

Em problemas de FPO estes erros numéricos podem ocorrer com mais frequência quando há sobrecargas de geração e/ou transmissão no sistema, pois variáveis de decisão atingem seus limites rapidamente. Além disso, tais sobrecargas podem ocasionar blackouts devido a incapacidade de satisfazer a demanda ou mesmo de transmitir a carga gerada, o que para estes métodos representa a não convergência. 
Em algumas situações, pode não ser possível evitar a ocorrência de sobrecargas. Assim, são necessárias ações que visam operar com o sistema sobrecarregado por um curto período de tempo, até que operações corretivas sejam tomadas. Nestes casos, podem-se relaxar algumas restrições de modo que estes pontos operacionais se tornem interiores.

Este trabalho propõe a aplicação da função barreira logarítmica modificada proposta por Polyak [5] para o estudo de viabilidade operacional de um problema de FPO CC com sobrecargas, tanto nas linhas de transmissão, quanto em unidades geradoras, visando manter o sistema operando de forma segura, sem a ocorrência de blackouts.

\section{Relaxando o Modelo de FPO Linearizado via Fluxo em Redes}

O modelo em interesse é de despacho de potência ativa com critério quadrático separável cuja formulação CC é feita via fluxo em redes como em $[2,4]$ :

$$
\begin{gathered}
\min \quad \frac{\alpha}{2} f^{t} R f+\frac{\beta}{2}\left(p^{t} Q p+c^{t} p\right) \\
\text { s.a } \\
\text { Af }=E p-l \\
X f=0 \\
f_{l} \leq f \leq f_{u} \\
p_{l} \leq p \leq p_{u}
\end{gathered}
$$

onde:

- $m, n$ e $g$ são os números de barras, linhas de transmissão e de geradores respectivamente;

- $Q$ e $R$ : Matrizes diagonais $g \times g$ da componente quadrática do custo de geração e $g \times 1$ da componente linear do custo de geração respectivamente;

- $c$ e $l$ : Vetores $g \times 1$ da componente linear do custo de geração e $m \times 1$ de demanda de potência ativa respectivamente;

- $A$ e $X$ : Matrizes $m \times n$ de incidência da rede de transmissão e $(n-m+1) \times n$ de reatância das linhas respectivamente;

- $f$ e $p$ : Vetores $n \times 1$ de fluxo e $g \times 1$ de geração de potência ativa respectivamente;

- E: matriz de ordem $m \times g$ com cada coluna contendo exatamente um elemento igual a 1 , correspondendo às barras de geração, e os demais elementos nulos;

- $f_{u}, f_{l}, p_{u}$ e $p_{l}$ : limites de fluxo e de geração de potência ativa respectivamente;

- $\alpha$ e $\beta$ : ponderações dos objetivos a minimizar.

O sistema de transmissão é representado por um fluxo de carga CC com limites no fluxo das linhas. Para que $f$ e $p$ possam ser expressas simultaneamente no modelo, as leis de Kirchhoff para nós e ramos (1) e (2) são apresentadas separadamente [2]. Portanto, o conjunto de restrições para este problema é linear onde, as equações (1) e (2) representam a rede de geração/transmissão e as equações (3) e (4) representam as capacidades de transmissão e de geração do sistema. Neste modelo, as duas componentes da função objetivo são quadráticas com variáveis separáveis, a primeira representando o valor econômico das perdas de transmissão e a segunda o custo de geração das usinas, tanto térmicas quanto hidrelétricas.

Como este problema não está na forma padrão, são feitas mudanças de variáveis afim de que os limites inferiores sejam iguais a zero, e além disso são acrescentadas variáveis de folga, obtendo a seguinte formulação primal na forma padrão: 
$\min$

$$
\alpha\left(\frac{1}{2} f^{t} R f+c_{f}^{t} f\right)+\beta\left(\frac{1}{2} p^{t} Q p+c_{p}^{t} p\right)
$$

S.a

$$
\begin{aligned}
B f-\widehat{E} p & =\widehat{l} \\
f+s_{f} & =f_{u} \\
p+s_{p} & =p_{u} \\
\left(f, p, s_{f}, s_{p}\right) & \geq 0
\end{aligned}
$$

onde

$$
\begin{aligned}
B=\left[\begin{array}{c}
A \\
X
\end{array}\right], & \widehat{E}=\left[\begin{array}{c}
E \\
0
\end{array}\right], \quad \widehat{l}=\left[\begin{array}{l}
l^{a} \\
l^{b}
\end{array}\right] \\
l^{a} & =E p_{l}-A f_{l}-l \\
l^{b} & =-X f_{l} \\
c_{f} & =R f_{l}
\end{aligned}
$$

Considerando o problema de (5), há situações operacionais de sobrecarga nas quais não é possível o atendimento de todas as restrições. Nestes casos são interessantes soluções que, na prática, possam ser despachadas por um curto período de tempo, não colocando o sistema geração/transmissão em risco.

A fim de manter a continuidade de fornecimento de energia para todos os consumidores, algumas restrições podem ser relaxadas [3]. Para estas situações de emergência, pode-se reformular o problema (5) relaxando algumas de suas restrições, expressando permissão de violações de limites operacionais. Estas violações podem ser modeladas matematicamente como uma relaxação das variáveis de folga $s_{p}$ e $s_{f}$ da forma:

$$
s_{p} \geq-\delta_{p}^{-1} \quad s_{f} \geq-\delta_{f}^{-1}
$$

onde $\delta_{p}>0$ e $\delta_{f}>0$ sejam suficientes para que $p>p_{u}$ e $f>f_{u}$ quando necessário.

Assim, $\delta_{f}^{-1} \geq 0$ e $\delta_{p}^{-1} \geq 0$ representam as violações permitidas na transmissão e na geração respectivamente, ou seja, a diferença entre os limites operacionais de curto e de longo prazo. Estas relaxações $\delta_{f}^{-1}$ e $\delta_{p}^{-1}$ devem respeitar alguns critérios, como por exemplo o quanto se pode transmitir acima dos limites das linhas sem que estas caiam, ou mesmo o quanto se pode exigir de potência acima dos limites de geração sem que os danos parem o sistema.

A relaxação (6) possibilita que componentes das variáveis $s_{p}$ e $s_{f}$ se tornem negativas, fazendo com que estas deixem de ser interiores, o que era de se esperar, visto que a solução do problema não está no interior da região factível. Porém, com esta relaxação o método seguidor de caminhos não pode ser utilizado, pois faz uso de barreira logarítmica clássica, impondo a positividade destas variáveis. Assim, devemos trocar a função barreira de modo que sua condição de existência contemple a relaxação (6). É neste ponto que recorremos a função barreira logarítmica modificada introduzida por Polyak [5], que para um problema da forma:

$$
\begin{array}{ll}
\text { Minimizar } & f(x) \\
\text { sujeito a } & g_{i}(x) \geq 0, \quad i=1,2, \ldots, m \\
& x \in \Omega
\end{array}
$$

onde $\Omega=\left\{x \in R^{n} \mid g_{i}(x) \geq 0\right\}$, a função barreira proposta é a seguinte:

$$
\mathcal{M}_{\mathcal{L}}(x, \pi)=\left\{\begin{array}{cc}
f(x)-\frac{1}{\delta} \sum_{i=1}^{m} \pi_{i} \ln \left(\delta g_{i}(x)+1\right) & , \text { se } x \in \Omega_{\delta} \\
\infty & \text {, se } x \notin \Omega_{\delta} .
\end{array}\right.
$$

onde $\Omega_{\delta}=\left\{x \mid \delta g_{i}(x)+1 \geq 0\right\}$.

Esta função barreira se encaixa perfeitamente no desejado, visto que a condição de existência do logarítmo é $g_{i}(x) \geq-\delta^{-1}$ e, trabalhando com o conjunto relaxado $\Omega_{\delta}$, é possível gerar pontos 
infactíveis em relação ao conjunto $\Omega$, mas nunca fora de $\Omega_{\delta}$. Observe ainda que quanto maior for o parâmetro $\delta$, menor é a expansão do conjunto $\Omega$.

Na literatura sobre métodos de barreira logarítmica modificada sugere-se que estes parâmetros de barreira sejam mantidos fixos ou aumentados a cada iteração. Porém, para este problema, aumentá-los a cada iteração levaria a uma redução das relaxações durante o processo iterativo. Assim sendo, deve-se ter um limitante para esse aumento de modo a respeitar a relaxação permitida, ou deixá-lo fixo, pois caso contrário, o problema em questão não será resolvido.

Neste trabalho vamos relaxadar todas as variáveis primais $\mathcal{P}=\left\{f, p, s_{f}, s_{p}\right\}$ do problema (5) para fazer uso de uma única função barreira.

Como a violação temporária permitida para geradores é percentualmente inferior a permitida para linhas, tem-se que ter relaxações diferentes para as variáveis relacionadas a geradores e a linhas de transmissão. Neste caso usa-se $\delta_{f}$ para as variáveis $f$ e $s_{f}$, e $\delta_{p}$ para as variáveis $p$ e $s_{p}$, obtendo o seguinte problema relaxado equivalente:

$$
\min
$$

$$
\alpha\left(\frac{1}{2} f^{t} R f+c_{f}^{t} f\right)+\beta\left(\frac{1}{2} p^{t} Q p+c_{p}^{t} p\right)
$$

s.a

$$
\begin{aligned}
B f-\widehat{E} p & =\widehat{l} \\
f+s_{f} & =f_{u} \\
p+s_{p} & =p_{u} \\
\left(f, s_{f}\right) & \geq-\delta_{f}^{-1} e_{f} \\
\left(p, s_{p}\right) & \geq-\delta_{p}^{-1} e_{p} .
\end{aligned}
$$

Onde $e_{f}$ e $e_{p}$ são vetores de uns, de dimensões apropriadas.

Deste modo, tem-se a seguinte função Lagrangiana:

$$
\mathcal{L}=\varphi\left(f, p, s_{f}, s_{p}\right)-\delta_{x}{ }^{-1} \sum_{x \in \mathcal{P}} \phi\left(x, \pi_{x}, \delta_{x}\right)-y^{t}(\widehat{l}-B f+\widehat{E} p)-w_{s f}^{t}\left(f_{u}-f-s_{f}\right)-w_{s p}^{t}\left(p_{u}-p-s_{p}\right)
$$

onde $\begin{aligned} \varphi\left(f, p, s_{f}, s_{p}\right) & =\alpha\left(\frac{1}{2} f^{t} R f+c_{f}^{t} f\right)+\beta\left(\frac{1}{2} p^{t} Q p+c_{p}^{t} p\right) \\ \phi\left(x, \pi_{x}, \delta_{x}\right) & =\sum_{i \in I} \pi_{x_{i}} \ln \left(\delta_{x} x_{i}+1\right)\end{aligned}$

Resultando nas seguintes condições de otimalidade para o problema modificado:

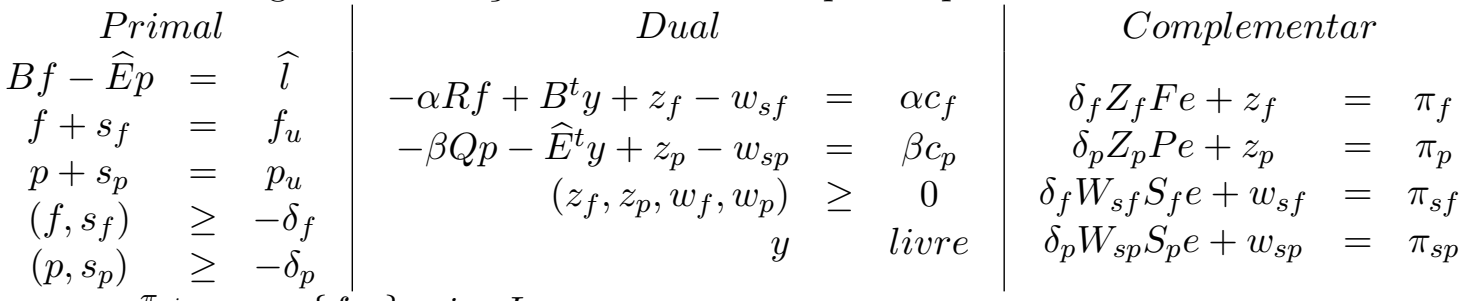

onde $z_{x i}=\frac{\pi_{x i}}{\left(\delta_{x} x_{i}+1\right)}, x \in\{f, p\}$ e $i \in I$.

Agora, basta aplicar o método de Newton nestas condições de otimalidade como feito no método seguidor de caminhos. Este modelo resolvido desta maneira obtém bons resultados não apenas quando há uma solução factível viável, mas também quando a soma dos limites de geração é menor que a demanda ou quando há congestionamento nas linhas de transmissão, nestes casos obtendo um despacho com sobrecargas.

\section{Controle de Passos}

Um ponto importante a ser analisado é o controle dos passos do método de Newton. Este controle não deve ser o mesmo utilizado no método seguidor de caminhos, visto que estes impedem que as variáveis se tornem negativas ou nulas.

Utilizando o mesmo raciocínio, pode-se definir um controle de passos eficiente para a abordagem proposta, permitindo que as componentes das variáveis com relaxação possam assumir valores no intervalo $\left(-\delta^{-1}, 0\right]$. A atualização deve ser da seguinte maneira: 


$$
x_{i}^{k}+\alpha d x_{i}^{k} \geq-\delta^{-1} .
$$

Logo, pode-se assumir $\alpha \geq-\left(x_{i}^{k}+\delta^{-1}\right) / d x_{i}^{k}$ para algum $i$.

Como $x^{k}$ é factível para o problema relaxado da iteração $k$, ou seja, $x_{i}^{k} \geq-\delta^{-1}$ segue que $x_{i}^{k}+\delta^{-1} \geq 0$. Portanto, assim como antes, deve-se preocupar apenas com as componentes $d x_{i}^{k}<0$. Deste modo, tem-se a seguinte expressão:

$$
\alpha=\min \left\{\left(-\left(x_{i}^{k}+\delta^{-1}\right) / d x_{i}^{k}, d x_{i}^{k}<0\right), 1\right\} .
$$

Para as demais variáveis fora da relaxação o controle de passos segue no modo tradicional.

\section{Estudo de Casos}

Os testes computacionais foram feitos em Sistema Operacional Ubuntu 12.4, 8GB de memória RAM com processadores Intel ${ }^{\circledR}$ Core $^{T M}$ i7-2670QM CPU $2.20 \mathrm{GHz}$ de 8 núcleos, utilizando o software Matlab R2012b.

Um estudo ilustrativo pode ser feito utilizando o sistema IEEE $30 \mathrm{com}$ as unidades geradoras $1,2,5,8,11$ e 13 cujas capacidades de geração são respectivamente $30 M W, 50 M W, 70 M W$, $70 M W, 40 M W$ e $40 M W$, ou seja, com potência instalada de $300 M W$ e uma carga de $283,4 M W$. Além disso, considerando os custos dos geradores 1 e 2 como sendo $1 U \$, 5$ e 8 sendo $4 U \$$ e 11 e 13 sendo $2 U \$$, com limites de cada linha de transmissão são fixados em $50 M W$.

Com o cenário descrito acima, o sistema se encontra em situação normal de operação, ou seja, sem sobrecargas. Os testes a seguir são realizados com os parâmetros de barreira $\alpha_{p}$ e $\alpha_{f}$ fixos. Em situações normais de operação, estes são definidos com valores muito grandes, para que a relaxação seja a menor possível, obtendo resultados compativeis com o método seguidor de caminhos. Para os casos de contingências, estes são definidos conforme as porcentagens de violações permitidas.

A Figura 1 mostra o diagrama unifilar deste sistema.

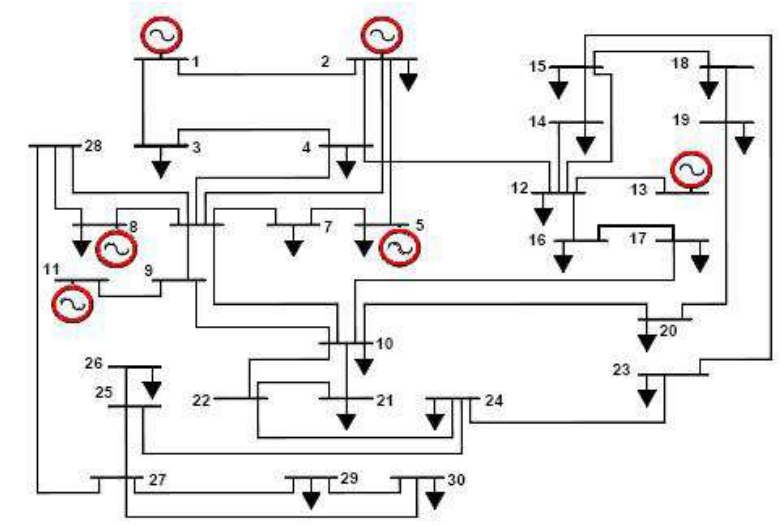

Figura 1: Diagrama Unifilar IEEE 30

Como na função objetivo do problema (5) há as ponderações $\alpha$ para transmissão e $\beta$ para geração, fazendo-se $\alpha=1$ e $\beta=0$ otimiza-se apenas as perdas na transmissão $(\mathbf{P t}), \alpha=0$ e $\beta=1$ otimiza-se apenas o custo na geração $(\mathbf{C g})$ e qualquer outro par de combinações positivas otimiza-se uma ponderação entre as perdas na transmissão e o custo na geração $(\mathbf{P t} \& \mathbf{C g})$.

Neste trabalho, as ponderações são dadas por $\alpha=c . m$ e $\beta=1$, onde $c . m$ representa o custo marginal dos geradores obtidos $\operatorname{com} \alpha=1$ e $\beta=0$. 


\subsection{Situações de Sobrecarga na Geração}

Simulando um novo cenário, a capacidade de geração da unidade 1 é reduzida de $30 M W$ para $10 M W$, reduzindo a capacidade de geração do sistema para $280 M W$, ou seja, faltando $3,4 M W$ para satisfazer a demanda.

A Tabela 1 exibe os despacho obtidos utilizando a formulação com relaxação em (9), com no máximo $10 \%$ de violação permitida em cada gerador e de no máximo $30 \%$ para cada linha.

\begin{tabular}{c|cc|cc|cc}
\hline Gerador & Pt (MW) & Violação(\%) & Cg (MW) & Violação(\%) & Pt \& Cg (MW) & Violação(\%) \\
\hline 1 & 10,69 & 6,86 & 10,02 & 0,17 & 10,00 & 0,00 \\
2 & 50,45 & 0,91 & 50,02 & 0,03 & 50,00 & 0,00 \\
5 & 70,27 & 0,38 & 69,77 & 0,00 & 71,80 & 2,57 \\
8 & 70,73 & 1,05 & 69,77 & 0,00 & 71,59 & 2,28 \\
11 & 40,36 & 0,91 & 41,82 & 4,55 & 40,01 & 0,01 \\
13 & 40,90 & 2,24 & 42,00 & 5,00 & 40,00 & 0,00 \\
\hline \multicolumn{2}{|c|}{8 iterações } & \multicolumn{2}{c|}{23 iterações } & \multicolumn{2}{c}{21 iterações } \\
\hline
\end{tabular}

Tabela 1: Despachos factíveis para o sistema com sobrecarga na geração.

Neste cenário houve apenas violação na geração, visto que esta não era suficiente para satisfazer a demanda do sistema. Observe na Tabela 1 que no despacho (Pt) todas as unidades geram acima de suas capacidades, mas há no máximo $6.86 \%$ de violação, justamente na unidade geradora 1 contingente.

Para o despacho $(\mathrm{Cg})$, por serem mais caras, as unidades 5 e 8 não sofreram violações, mas as unidades 11 e 13, mesmo sendo mais caras que as unidades 1 e 2 , sofrem maior violação.

Para o despacho (Cg \& Pt), apenas as unidades 5, 8 e 11 geram acima de seus limites, sendo o despacho que menos viola restrições.

Neste cenário o método seguidor de caminhos não converge, ou seja, os ultiplicadores de lagrange associados a geração tendem ao infinito, não fornecendo nenhum tipo de informação sobre onde e quanto investir no sistema. Já com a metodo proposto podemos continuar retirando informações dos multiplicadores de lagrange, conforme pode ser observado na Tabela 2:

\begin{tabular}{c|ccc}
\hline Gerador & Pt $(\mathrm{U} \$ / \mathrm{MW})$ & $\mathrm{Cg}(\mathrm{U} \$ / \mathrm{MW})$ & $\mathrm{Pt} \& \mathrm{Cg}(\mathrm{U} \$ / \mathrm{MW})$ \\
\hline 1 & 4,69 & $1,69 \times 10^{2}$ & $2,77 \times 10^{2}$ \\
2 & 4,78 & $2,29 \times 10^{2}$ & $2,37 \times 10^{2}$ \\
5 & 5,27 & $2,61 \times 10^{-4}$ & $1,00 \times 10^{-3}$ \\
8 & 4,43 & $2,97 \times 10^{-4}$ & $2,79 \times 10^{-4}$ \\
11 & 3,74 & $1,95 \times 10^{2}$ & $2,06 \times 10^{2}$ \\
13 & 4,09 & $1,95 \times 10^{2}$ & $2,06 \times 10^{2}$ \\
\hline
\end{tabular}

Tabela 2: Sensibilidade da função objetivo com relação à geração.

Nestas situações, o método proposto leva extrema vantagem sobre o seguidor de caminhos, pois além de obter soluções viáveis dentro dos limites operacionais de curto prazo, ainda fornece informações para planejamento e expansão do sistema.

\subsection{Situações de Sobrecarga na Transmissão}

No próximo estudo de casos, é forçada uma contingência nas linhas de transmissão, configurando uma incapacidade para transmitir a potência necessária para satisfazer a demanda, resultando em um congestionamento. Para isso podemos simular uma queda parcial da linha de transmissão que liga as unidades geradoras 2 e 5, visto que esta linha apresenta um fluxo alto. Reduzindo o limite de transmissão para $23 M W$, o método de pontos interiores novamente não converge devido 
a incapacidade de transmissão da linha 2-5, porém, o método proposto fornece informações valiosas por poder violar esta linha. Os despachos podem ser vistos na Tabela 3.

\begin{tabular}{c|ccc}
\hline Gerador & Pt (MW) & Cg (MW) & Pt \& Cg (MW) \\
\hline 1 & 30,00 & 30,00 & 30,00 \\
2 & 33,51 & 50,00 & 50,00 \\
5 & 70,00 & 70,00 & 70,00 \\
8 & 70,00 & 53,40 & 53,40 \\
11 & 39,89 & 40,00 & 40,00 \\
13 & 40,00 & 40,00 & 40,00 \\
\hline & 18 iterações & 17 iterações & 17 iterações \\
\hline
\end{tabular}

Tabela 3: Despachos com redução de capacidade da linha 2-5.

A unidade geradora 2 teve sua geração reduzida no despacho $(\mathrm{Pt})$ devido a incapacidade de uma de suas linhas, ocasionando um aumento no valor da função objetivo e uma violação de 6, 93\% na linha 2-5. A componente do multiplicador de Lagrange $w_{f}$ referente a esta linha ficou próximo de $30 U \$ / M W$ e os demais permaneceram próximos a zero.

Os despachos $(\mathrm{Cg})$ e $(\mathrm{Cg} \& \mathrm{Pt})$ foram iguais, diferenciando apenas na distribuição dos fluxos nas linhas. Em ambos, a violação na linha $2-5$ foi de 18,7\% levando o multiplicador de Lagrange associado a esta muito próximo de $600 U \$ / M W$.

Deste modo, o método proposto consegue obter um despacho que satisfaz a carga do sistema, mesmo sobrecarregado, mas dentro dos limites de segurança.

\section{Conclusões}

Os testes foram bem promissores, tanto na geração quanto na transmissão, obtendo despachos que satisfazem as demandas, não ocasionando blackouts no sistema quando fora de situações normais de operação.

O método proposto fornece informações sobre possíveis melhorias no sistema, mesmo quando há sobrecargas no sistema, através do estudo de seus multiplicadores de Lagrange, que não tendem ao infinito como ocorre nos métodos de pontos interiores.

A não convergência do método esteve ligada a escolha do parâmetro de barreira e sua atualização, ou ao ajuste de passos primais e duais.

\section{Referências}

[1] A. Azevedo, C. Castro, A. Oliveira, and S. Soares. Security constrained optimal active power flow via network model and interior point method. SBA Controle 83 Automacao, 20(2):206-216, 2009.

[2] M. F. Carvalho, S. Soares, and T. Ohishi. Optimal active power dispatch by network flow approach. IEEE Transactions on Power Systems, 3(3):1640-1647, 1988.

[3] Y. Y Hsu and H. C Kuo. A heuristic based fuzzy reasoning approach for distribution system service restoration. IEEE Transactions on Power Delivery, 9(2), 1994.

[4] A. R. L Oliveira, S. Soares, and L. Nepomuceno. Optimal active power dispatch combining network flow and interior point approaches. IEEE Transactions on Power Systems, 18(4):1235-1240, November 2003.

[5] R. Polyak. Modified barrier functions in linear programming. Department of Mathematical Sciences - IBM T.J. Watson Research Center, 1992. 